\title{
Enzymatic Formation of Indole-Containing Unnatural Cyclic Polyprenoids by Bacterial Squalene:Hopene Cyclase
}

\begin{abstract}
Hideya Tanaka, ${ }^{\dagger}$ Hiroshi Noguchi, $\dagger$ and Ikuro Abe*, $\uparrow, \mathfrak{I}$
$\dagger$ School of Pharmaceutical Sciences and the COE 21 Program, University of Shizuoka, Shizuoka 422-8526, Japan, and PRESTO, Japan Science and Technology Agency, Kawaguchi, Saitama 332-0012, Japan
\end{abstract}

3-((2E,6E,10E)-3,7,11,15-tetramethylhexadeca-2,6,10,14-tetraenyl)-1H-indole (4): ${ }^{1} \mathrm{H}$ NMR $\left(400 \mathrm{MHz}, \mathrm{CDCl}_{3}\right): \delta 7.87$ (brs, $\left.1 \mathrm{H}\right), 7.61(\mathrm{dtd}, 1 \mathrm{H}, J=7.8,1.1,0.9 \mathrm{~Hz}), 7.35(\mathrm{dt}, 1 \mathrm{H}, J=8.3,0.9$ Hz), $7.20(\mathrm{ddd}, 1 \mathrm{H}, J=8.2,7.0,1.1 \mathrm{~Hz}), 7.13(\mathrm{ddd}, 1 \mathrm{H}, J=7.9,7.0,1.1 \mathrm{~Hz}), 6.95(\mathrm{dt}, 1 \mathrm{H}, J=2.3$, $1.0 \mathrm{~Hz}), 5.48(\mathrm{dd}, 1 \mathrm{H}, J=7.8,6.8 \mathrm{~Hz}), 5.28(\mathrm{~m}, 3 \mathrm{H}), 3.48(\mathrm{~d}, 2 \mathrm{H}, J=6.8 \mathrm{~Hz}), 2.09(\mathrm{~m}, 12 \mathrm{H}), 1.78(\mathrm{~s}$, 3H, Me-17), 1.70 (s, 3H, Me-18), 1.62 (s, 6H, Me-19, 20), 1.61 (s, 3H, Me-21). ${ }^{13} \mathrm{C}$ NMR (100 $\left.\mathrm{MHz}, \mathrm{CDCl}_{3}\right): \delta 136.5,135.6,135.0,134.9,131.2,127.5,124.4,124.3,123.6,122.9,121.9,121.1$, 119.1, 119.0, 116.1, 111.0, 39.7, 26.8, 26.7, 26.6, 25.7, 24.0, 17.7, 16.1, 16.0, 16.0. HRMS (FAB): found for $\left[\mathrm{C}_{28} \mathrm{H}_{39} \mathrm{~N}\right]^{+}$389.3059; calcd. 389.3082 .

3-((2E,6E,10E)-2,7,11,15-tetramethylhexadeca-2,6,10,14-tetraenyl)- $1 H$-indole $(5):{ }^{1} \mathrm{H} \quad \mathrm{NMR}$ $\left(400 \mathrm{MHz}, \mathrm{CDCl}_{3}\right): \delta 7.91$ (brs, $\left.1 \mathrm{H}\right), 7.62(\mathrm{ddt}, 1 \mathrm{H}, J=7.8,1.1,0.9 \mathrm{~Hz}), 7.34(\mathrm{dt}, 1 \mathrm{H}, J=8.3,0.9$ $\mathrm{Hz}), 7.18(\mathrm{ddd}, 1 \mathrm{H}, J=8.2,7.0,1.2 \mathrm{~Hz}), 7.10(\mathrm{ddd}, 1 \mathrm{H}, J=7.9,7.0,1.1 \mathrm{~Hz}), 6.97(\mathrm{dt}, 1 \mathrm{H}, 2.3,1.1)$, $5.39(\mathrm{~m}, 1 \mathrm{H}), 5.14(\mathrm{~m}, 3 \mathrm{H}), 3.46(\mathrm{~s}, 2 \mathrm{H}), 2.04(\mathrm{~m}, 12 \mathrm{H}), 1.70$ (s, 3H, Me-17), 1.63 (s, 3H, Me-18), 1.62 (s, 9H, Me-19, 20, 21). ${ }^{13} \mathrm{C}$ NMR (100 MHz, $\left.\mathrm{CDCl}_{3}\right): \delta 136.4,135.1,134.9,134.2,131.2$, $127.9,125.4,124.4,124.3,122.0,121.8,119.4,119.1,114.8,110.9,39.8,39.7,35.8,28.4,28.2$, 26.8, 26.7, 25.7, 17.7, 16.1, 16.0. HRMS (FAB): found for $\left[\mathrm{C}_{28} \mathrm{H}_{39} \mathrm{~N}\right]^{+}$389.3094; calcd. 389.3082. 
Major product (6): ${ }^{1} \mathrm{H}$ NMR (500 MHz, $\mathrm{CDCl}_{3}$ ): $\delta 7.92$ (brs, $\left.1 \mathrm{H}, \mathrm{NH}\right), 7.64(\mathrm{dt}, 1 \mathrm{H}, J=7.9$, $1.1 \mathrm{~Hz}), 7.36(\mathrm{dt}, 1 \mathrm{H}, J=8.0,1.0 \mathrm{~Hz}), 7.19(\mathrm{ddd}, 1 \mathrm{H}, J=8.0,7.0,1.1 \mathrm{~Hz}), 7.12(\mathrm{ddd}, 1 \mathrm{H}, J=8.0$, 7.0, $1.0 \mathrm{~Hz}), 6.98(\mathrm{~d}, 1 \mathrm{H}, J=1.7 \mathrm{~Hz}), 5.19(\mathrm{~d}, 1 \mathrm{H}, J=2.3 \mathrm{~Hz}), 3.07(\mathrm{dd}, 1 \mathrm{H}, J=14.0,2.6 \mathrm{~Hz}), 2.77$ (brd, 1H), $2.36(1 \mathrm{H}), 2.36(1 \mathrm{H}) 1.10$ (s, 3H, Me-18), 0.92 (s, 3H, Me-17), 0.88 (s, 3H, Me-16), 0.88 (s, 3H, Me-19), 0.78 (d, 3H, $J=6.9 \mathrm{~Hz}, \mathrm{Me}-20) .{ }^{13} \mathrm{C}$ NMR (125 MHz, $\left.\mathrm{CDCl}_{3}\right): \delta 158.4(\mathrm{C}-9), 136.4$ (C-8'), 127.8 (C-9'), 121.7 (C-2'), 121.7 (C-6'), 119.1 (C-5'), 119.0 (C-4'), $116.4\left(\mathrm{C}-3^{\prime}\right), 115.5$ (C-11), 111.0 (C-7'), 50.26 (C-8), 48.2 (C-13), 47.0 (C-12), 45.7 (C-5), 44.6 (C-14), 42.6 (C-3), 40.1 (C-1), 36.4 (C-10), 33.4 (C-4), 33.0 (C-16), 28.4 (C-15), 25.1 (C-18), 22.1 (C-7), 21.5 (C-17), 19.4 (C-5), 19.2 (C-2), 17.0 (C-19), 15.7 (C-20). The NMR assignments were performed according to data from H-H COSY, HMQC, HMBC, NOESY, differential NOE experiments. LRMS (FAB $\left.{ }^{+}, \%\right): m / z$ 130 (98), 158 (67), $231(100), 390\left(\mathrm{M}^{+}, 15\right)$. HRMS $\left(\mathrm{FAB}^{+}\right)$: found for $\left[\mathrm{C}_{28} \mathrm{H}_{39} \mathrm{~N}\right]$ 389.3059; calcd. 389.3082. $[\alpha]_{\mathrm{D}}^{25}=-6.7^{\circ}\left(\mathrm{c}=0.10\right.$ in $\left.\mathrm{CHCl}_{3}\right)$.
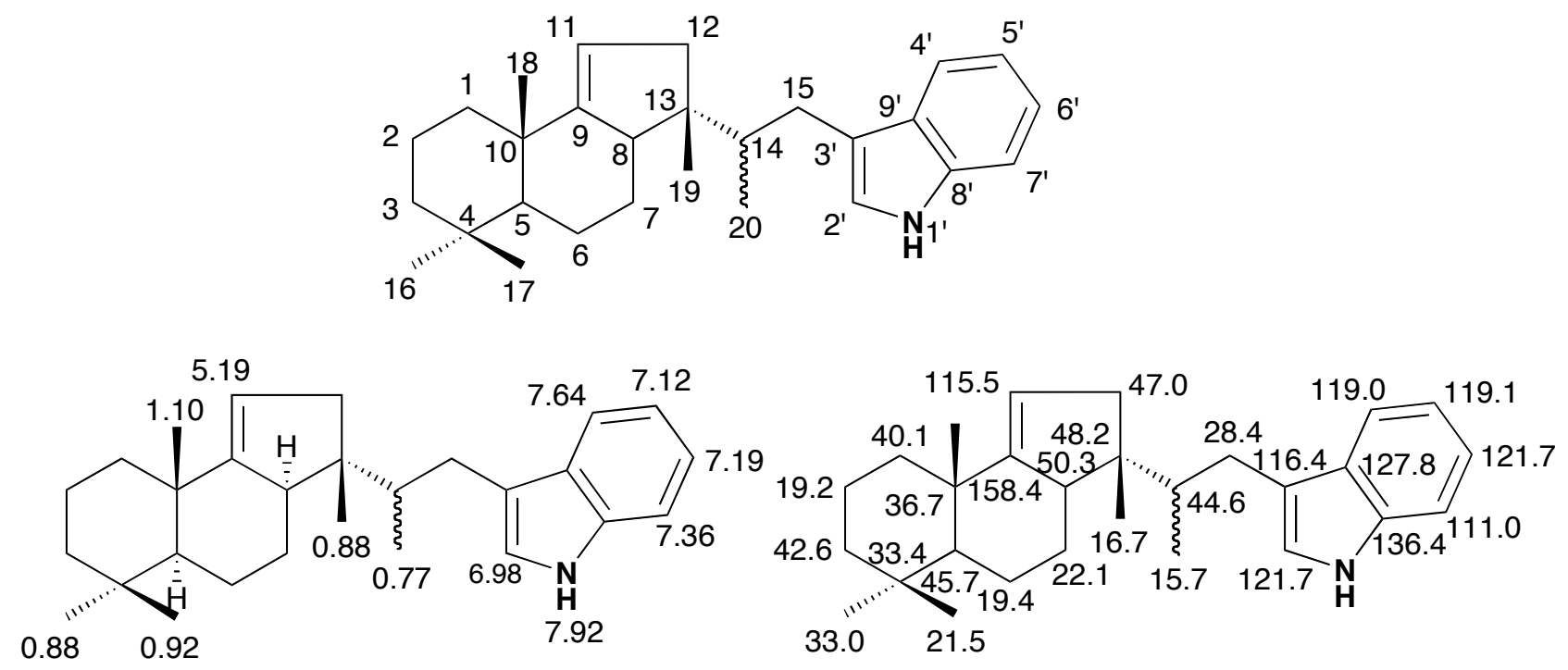
Minor product (7): ${ }^{1} \mathrm{H}$ NMR $\left(500 \mathrm{MHz}, \mathrm{CDCl}_{3}\right): \delta 7.93$ (brs, $\left.1 \mathrm{H}, \mathrm{NH}\right), 7.62(\mathrm{dt}, 1 \mathrm{H}, J=8.0$, $1.1 \mathrm{~Hz}), 7.35(\mathrm{dt}, 1 \mathrm{H}, J=8.2,1.0 \mathrm{~Hz}), 7.17(\mathrm{ddd}, 1 \mathrm{H}, J=8.2,7.0,1.2 \mathrm{~Hz}), 7.09$ (ddd, 3H, $J=8.0$, 7.0, $1.2 \mathrm{~Hz}), 6.97(\mathrm{t}, 1 \mathrm{H}, J=1.1 \mathrm{~Hz}), 5.43(\mathrm{~m}, 1 \mathrm{H}), 5.34(\mathrm{~m}, 1 \mathrm{H}), 3.44(\mathrm{~s}, 2 \mathrm{H}, \mathrm{Me}-30), 1.95(\mathrm{~m}, 2 \mathrm{H})$, 1.63 (s, 3H, Me-34), 1.55 (s, 3H, Me-34), 1.06 (s, 3H, Me-17), 1.00 (s, 3H, Me-16), 0.83 (d, 3H, J $=6.8 \mathrm{~Hz}, \mathrm{Me}-19), 0.61$ (s, 3H, Me-18). ${ }^{13} \mathrm{C} \mathrm{NMR}\left(125 \mathrm{MHz}, \mathrm{CDCl}_{3}\right): \delta 146.3(\mathrm{C}-5), 133.6(\mathrm{C}-14)$, 133.6 (C-8'), 126.1 (C-13), 126.0 (C-9'), 122.1 (C-9), 122.1 (C-6'), 121.8 (C-2'), 119.4 (C-4'), 119.1 (C-5'), 116.1 (C-6), 114.9 (C-3'), 110.9 (C-7'), 41.0 (C-3), 39.9 (C-10), 39.9 (C-10), 37.1 (C-15), 36.7 (C-11), 35.8 (C-4), 35.8 (C-9), 35.8 (C-15), 33.5 (C-8), 31.7 (C-7), 29.8 (C-17), 29.0 (C-16), 27.6 (C-1), 22.3 (C-2), 21.5 (C-12), 16.1 (C-18), 15.9 (C-20), 15.2 (C-19). The NMR assignments were performed according to data from H-H COSY, HMQC, HMBC, differential NOE experiments. LRMS (FAB $\left.{ }^{+}, \%\right): m / z 130(100), 184(48), 390\left(\mathrm{M}^{+}, 30\right)$. HRMS $\left(\mathrm{FAB}^{+}\right)$: found for $\left[\mathrm{C}_{28} \mathrm{H}_{39} \mathrm{~N}\right]$ 389.3059; calcd. 389.3082. $[\alpha]_{\mathrm{D}}^{25}=0.1^{\circ}\left(\mathrm{c}=0.10\right.$ in $\left.\mathrm{CHCl}_{3}\right)$.
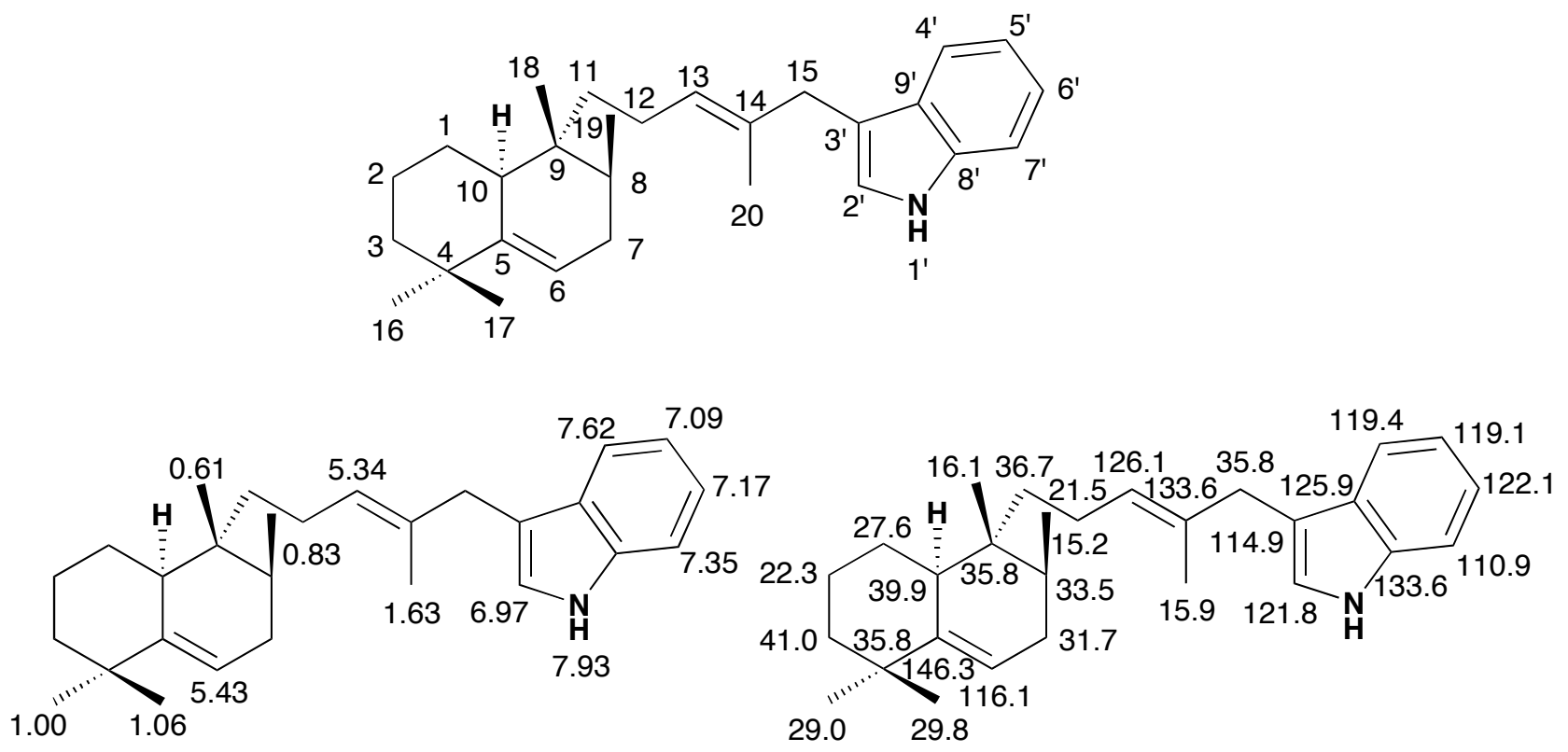
${ }^{1} \mathrm{H} /{ }^{13} \mathrm{C}$ NMR of 3-((2E,6E,10E)-3,7,11,15-tetramethylhexadeca-2,6,10,14-tetraenyl)-1H-indole (4)
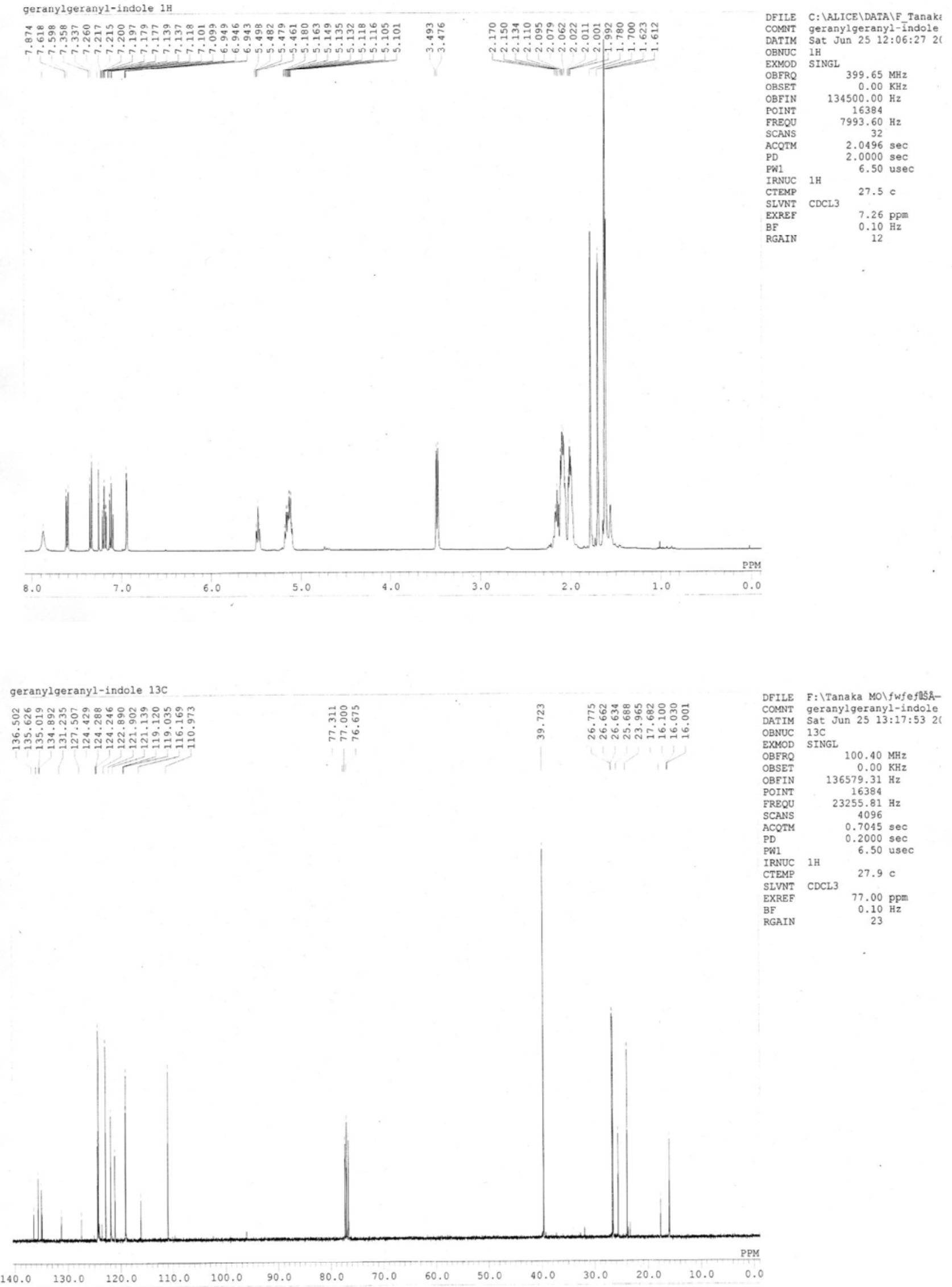
${ }^{1} \mathrm{H} /{ }^{13} \mathrm{C}$ NMR of 3-((2E,6E,10E)-2,7,11,15-tetramethylhexadeca-2,6,10,14-tetraenyl)- $1 H$-indole (5)

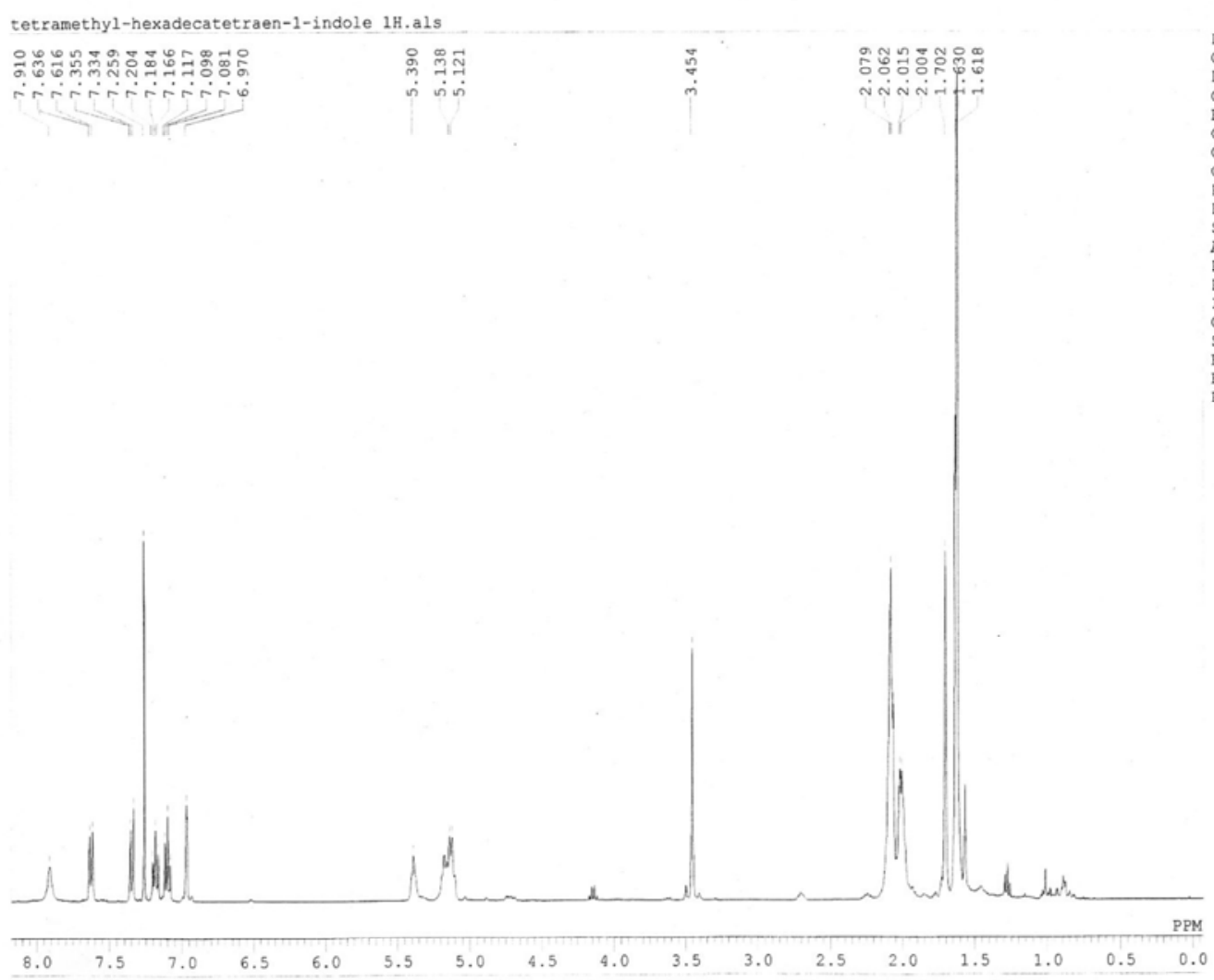

DFILE F: \Tanaka MO\fwfeflSÁ-

COMNT tetramethyl-hexadecat

DANUC $1 \mathrm{H}$

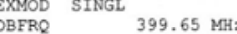

$\begin{array}{lrl}399.65 \mathrm{MH} \\ \text { OBSET } & 0.00 \mathrm{KH}\end{array}$

OBFIN $\quad 134500.00 \mathrm{~Hz}$

$\begin{array}{ll} & 16384 \\ \text { FREQU } & 7993.60 \mathrm{~Hz}\end{array}$

SCANS 128

PD $\quad 2.0000 \mathrm{sec}$

PW1 6.50 use

CTEMP $25.3 \mathrm{C}$

SLVNT CDCL3

$\begin{array}{ll}\text { EXREF } & 7.26 \mathrm{ppm} \\ \mathrm{BF} & 0.10 \mathrm{~Hz}\end{array}$

$\begin{array}{llllllllllllllll}7.5 & 7.0 & 6.5 & 6.0 & 5.5 & 5.0 & 4.5 & 4.0 & 3.5 & 3.0 & 2.5 & 2.0 & 1.5 & 1.0 & 0.5 & 0.0\end{array}$

tetramethyl-hexadecatetraen-1-indole 13c.als

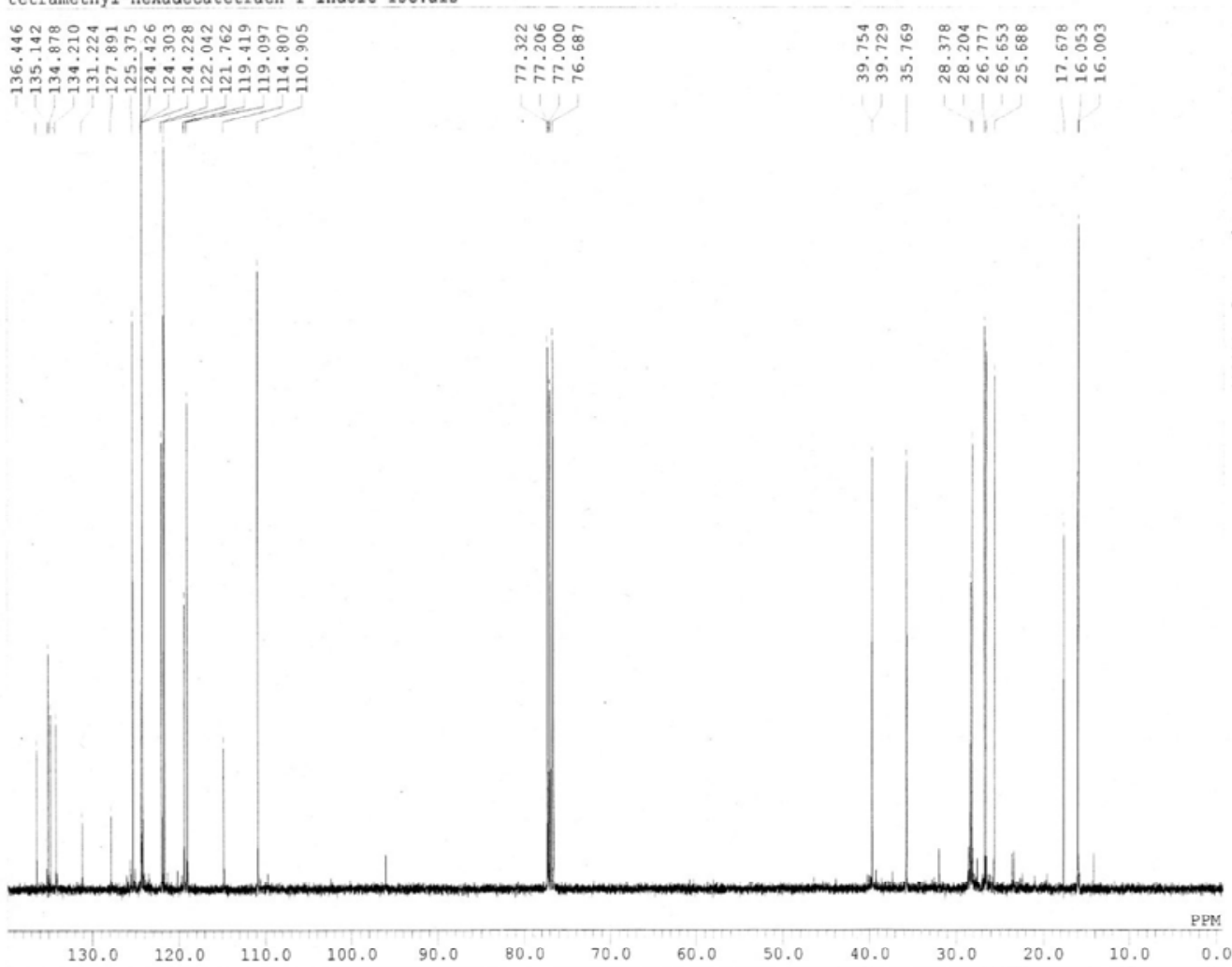

DFILE F: TTanaka MO\fwfeftŠA

COMNT Tetramethyl-hexadecat

Sat Jun 18 11:41:41 26

BNUC $13 \mathrm{C}$

OBFRQ $\quad 100.40 \mathrm{MHz}$

OBSET $\quad 0.00 \mathrm{KH}$

135500.00
32768

FREQU $27173.91 \mathrm{~Hz}$

SCANS $\quad 2048$

ACANM $\quad 1.2059 \mathrm{sec}$

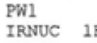

6.50 usec

SLVNT CDCL3

BE

7.00 ppm

$77.00 \mathrm{ppm}$
$0.10 \mathrm{~Hz}$ 
Major product (6): ${ }^{1} \mathrm{H} /{ }^{13} \mathrm{C}$ NMR
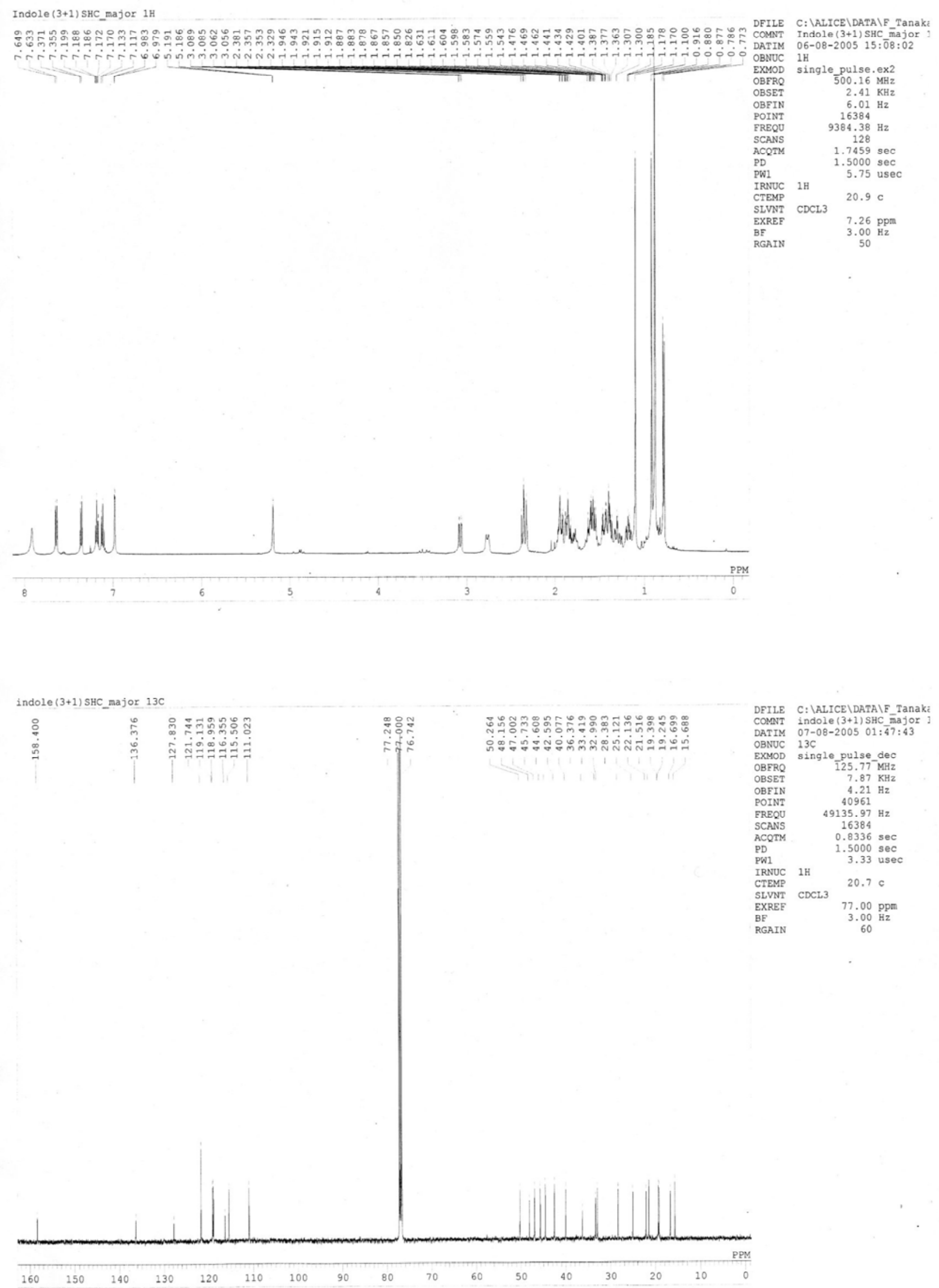
Major product (6): HMQC \& HMBC
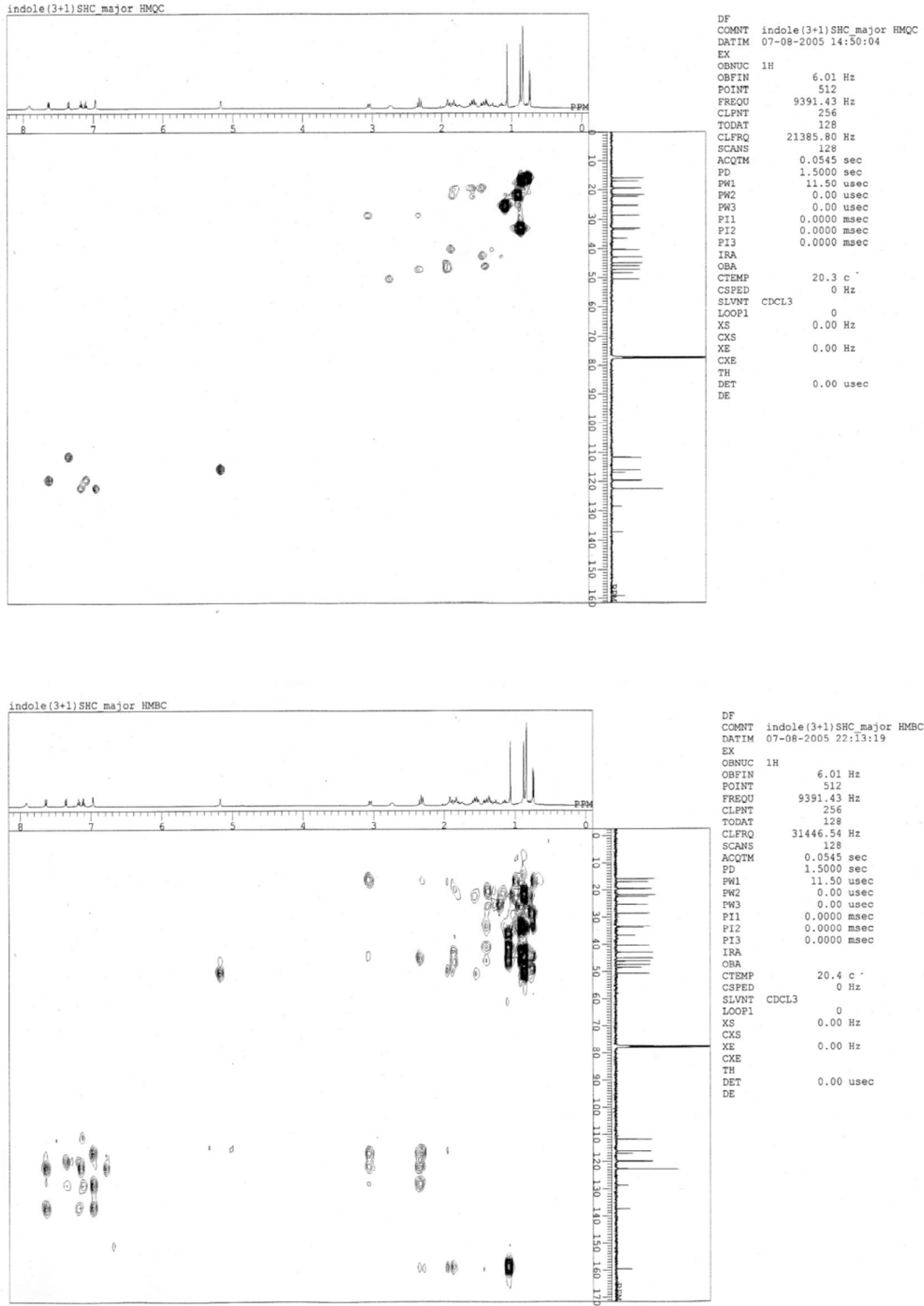
Major product (6): NOE Experiment-1

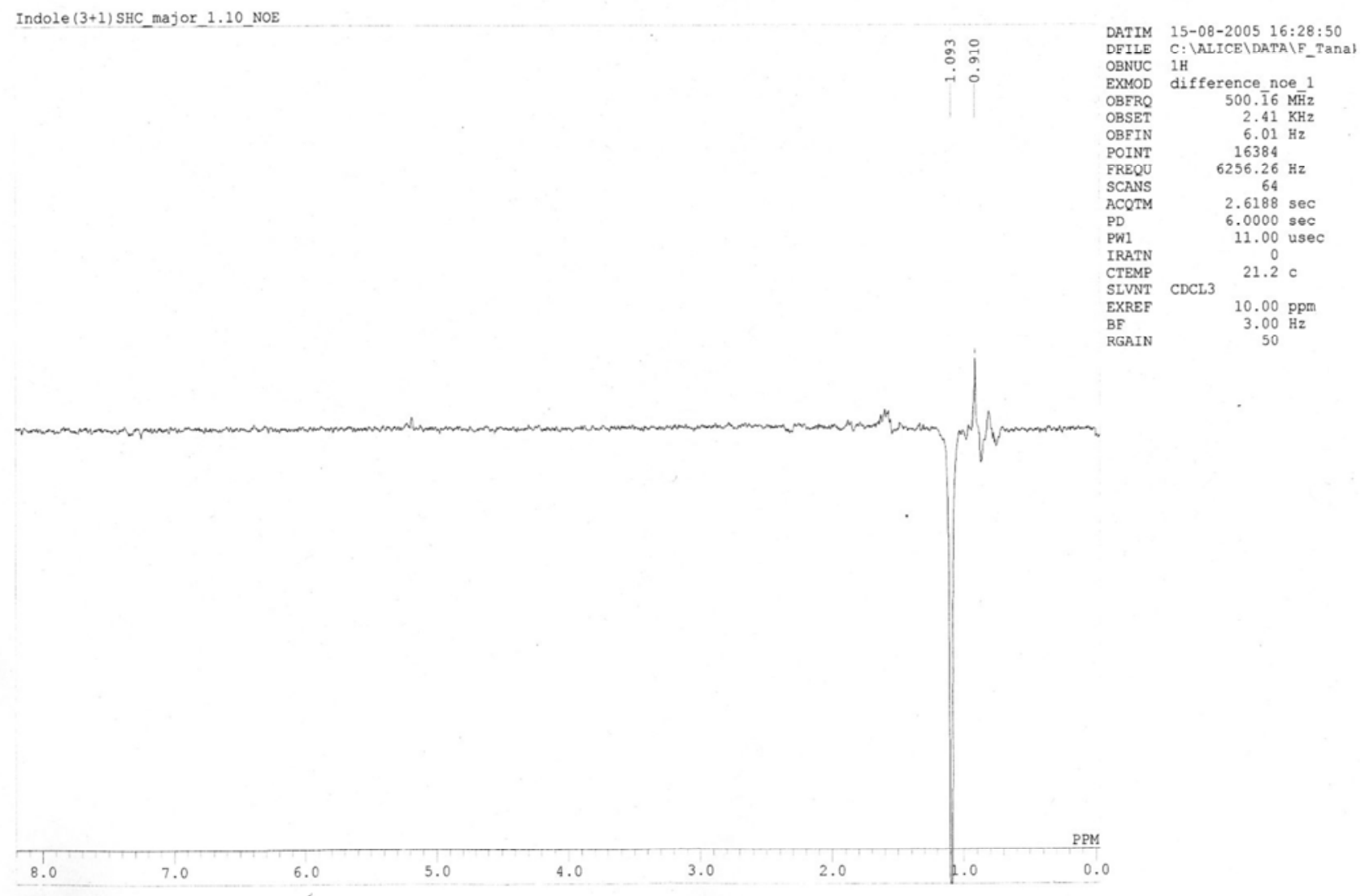

indole(3+1)SHC_RT21.9_0.77 NOE

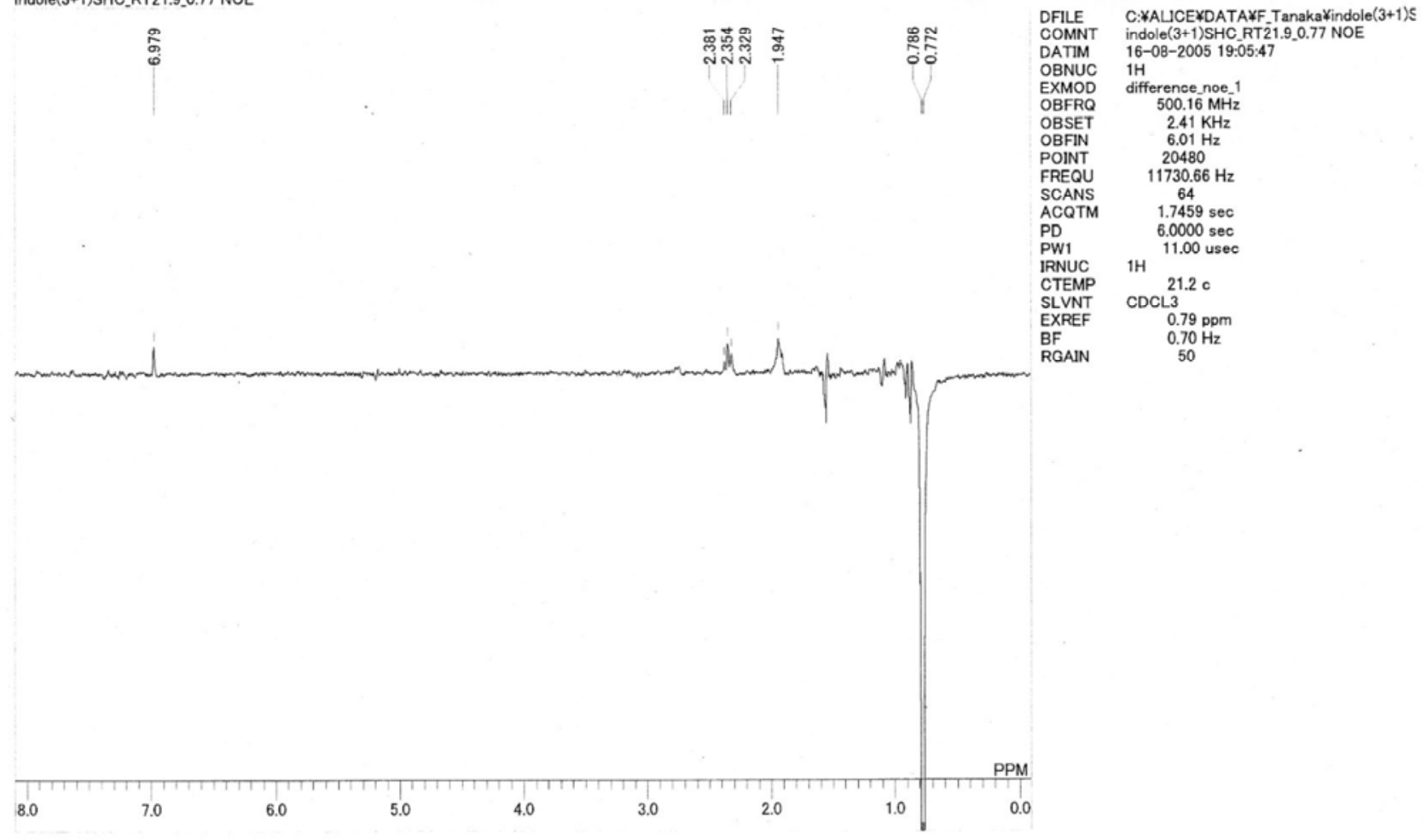


Major product (6): NOE Experiment-2

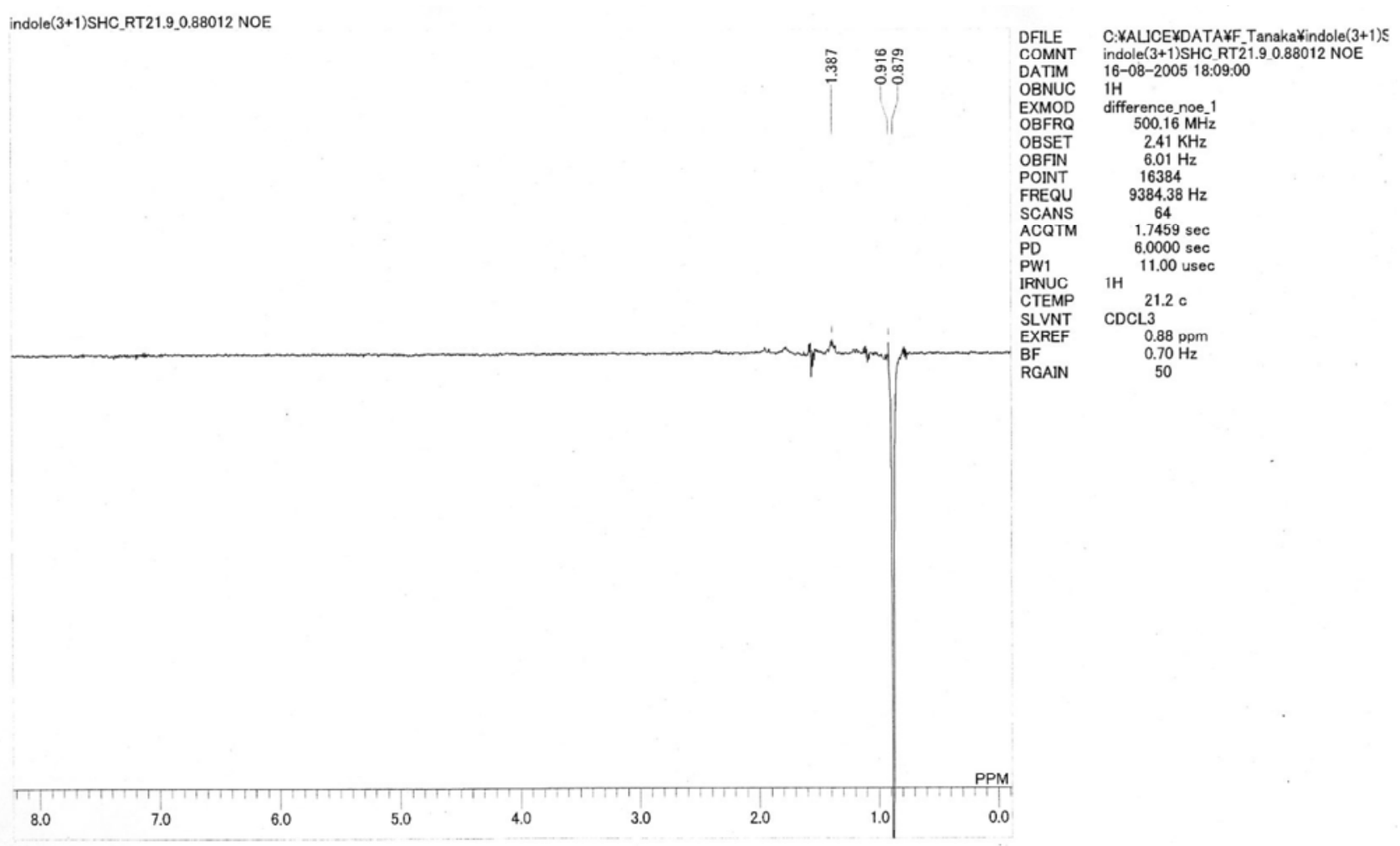

indole(3+1)SHC_RT21.9_0.91561_NOE

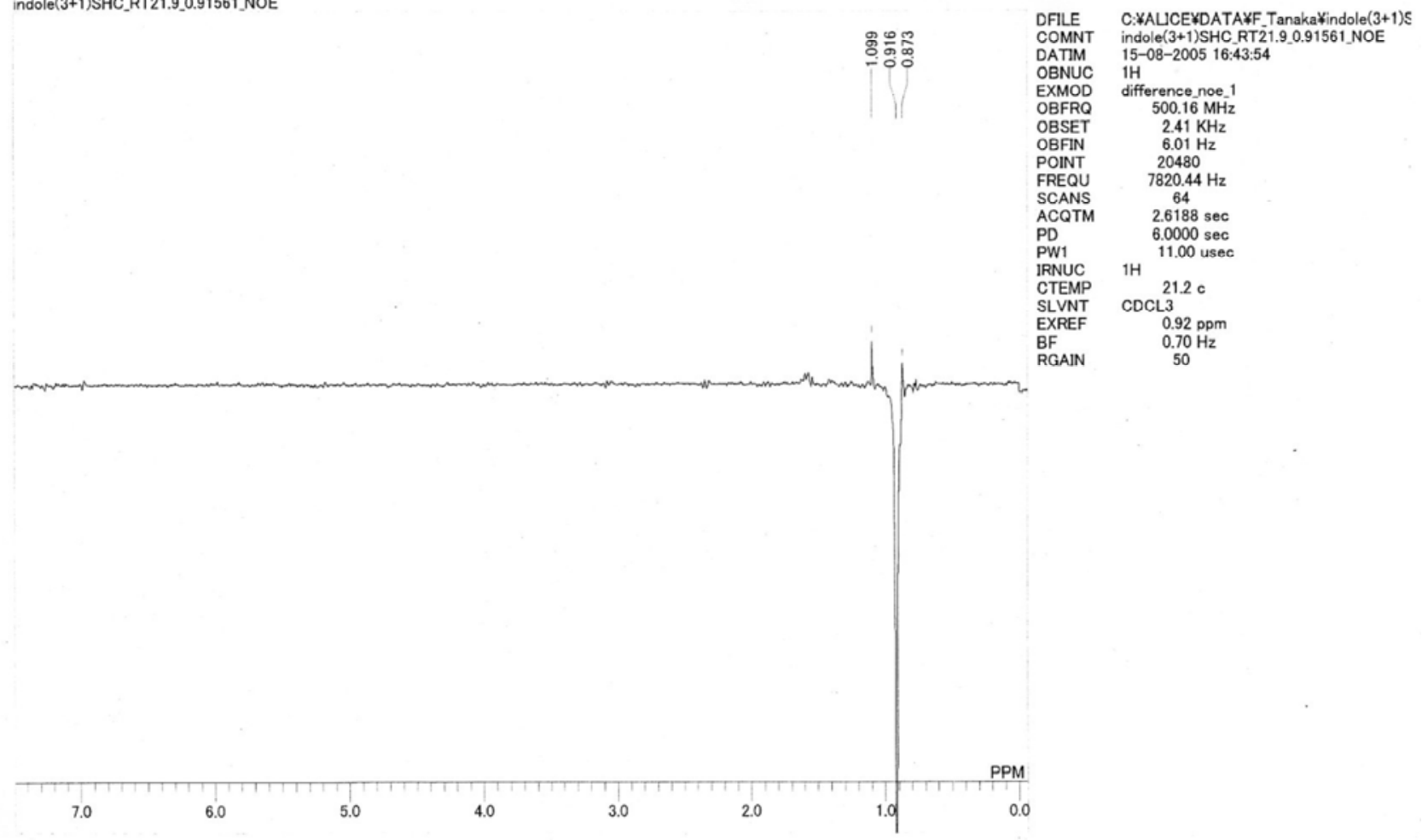


Major product (6): NOE Experiment-3

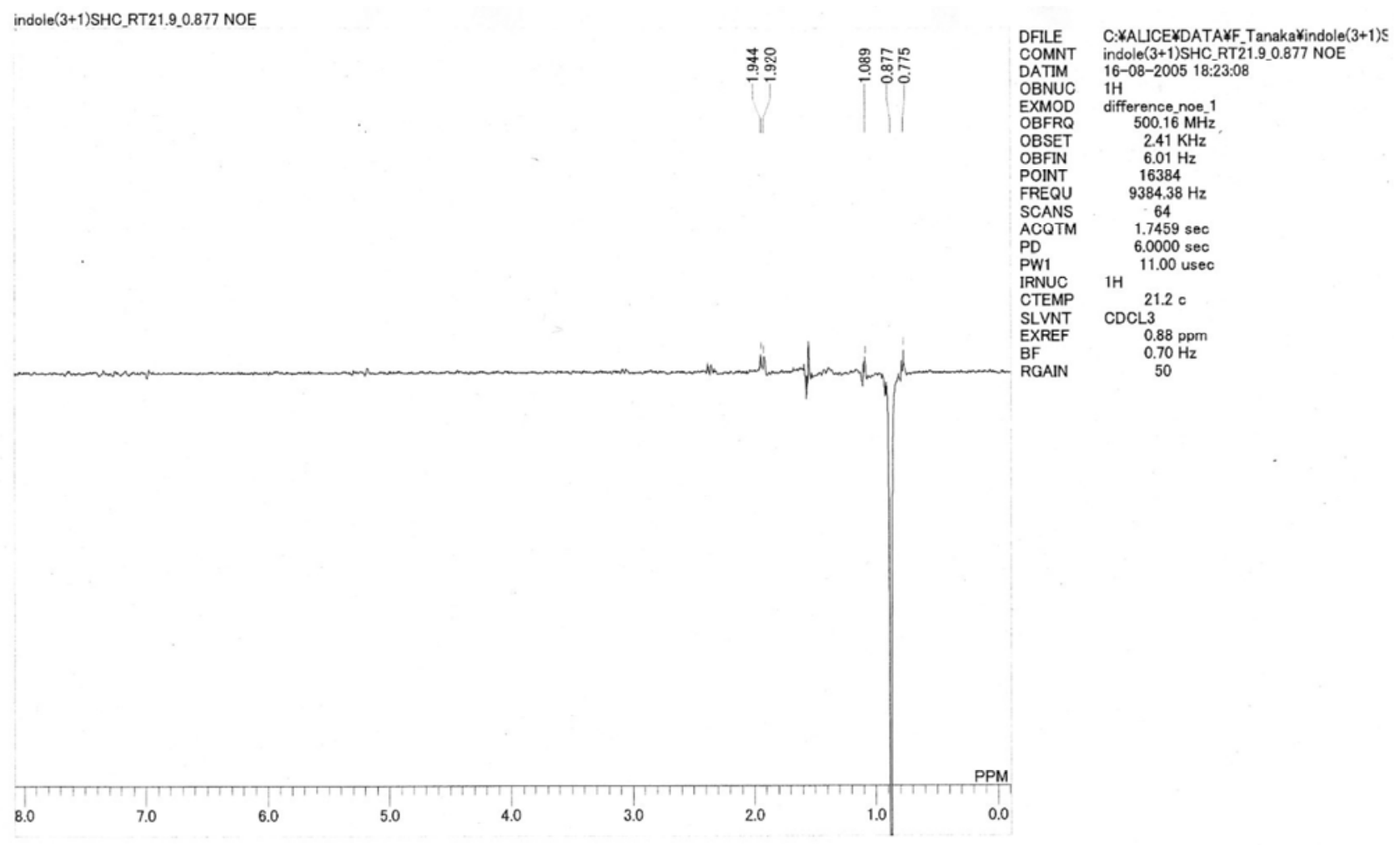


Minor product (7): ${ }^{1} \mathrm{H} /{ }^{13} \mathrm{C}$ NMR

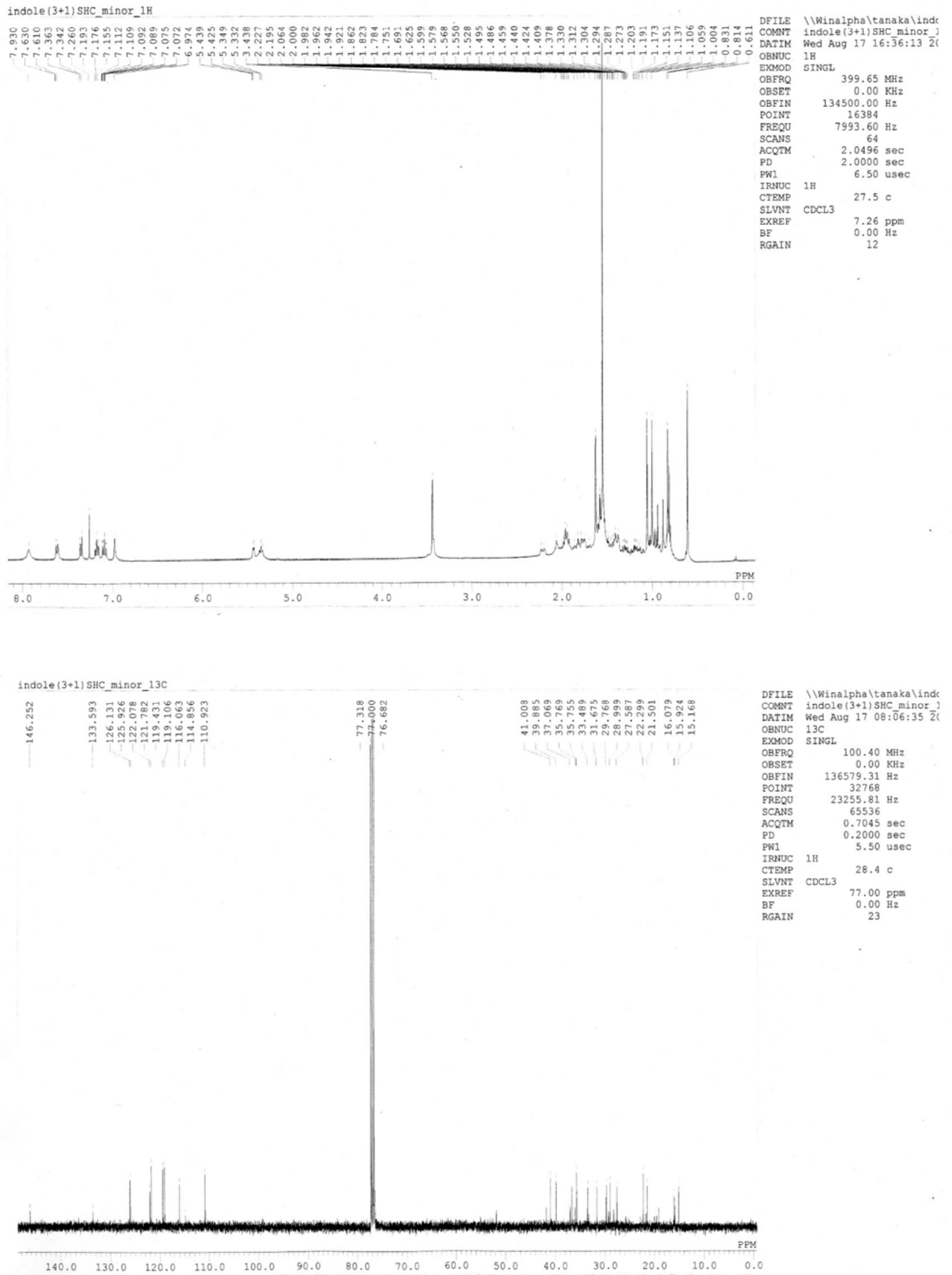


Minor product (7): HMQC \& HMBC
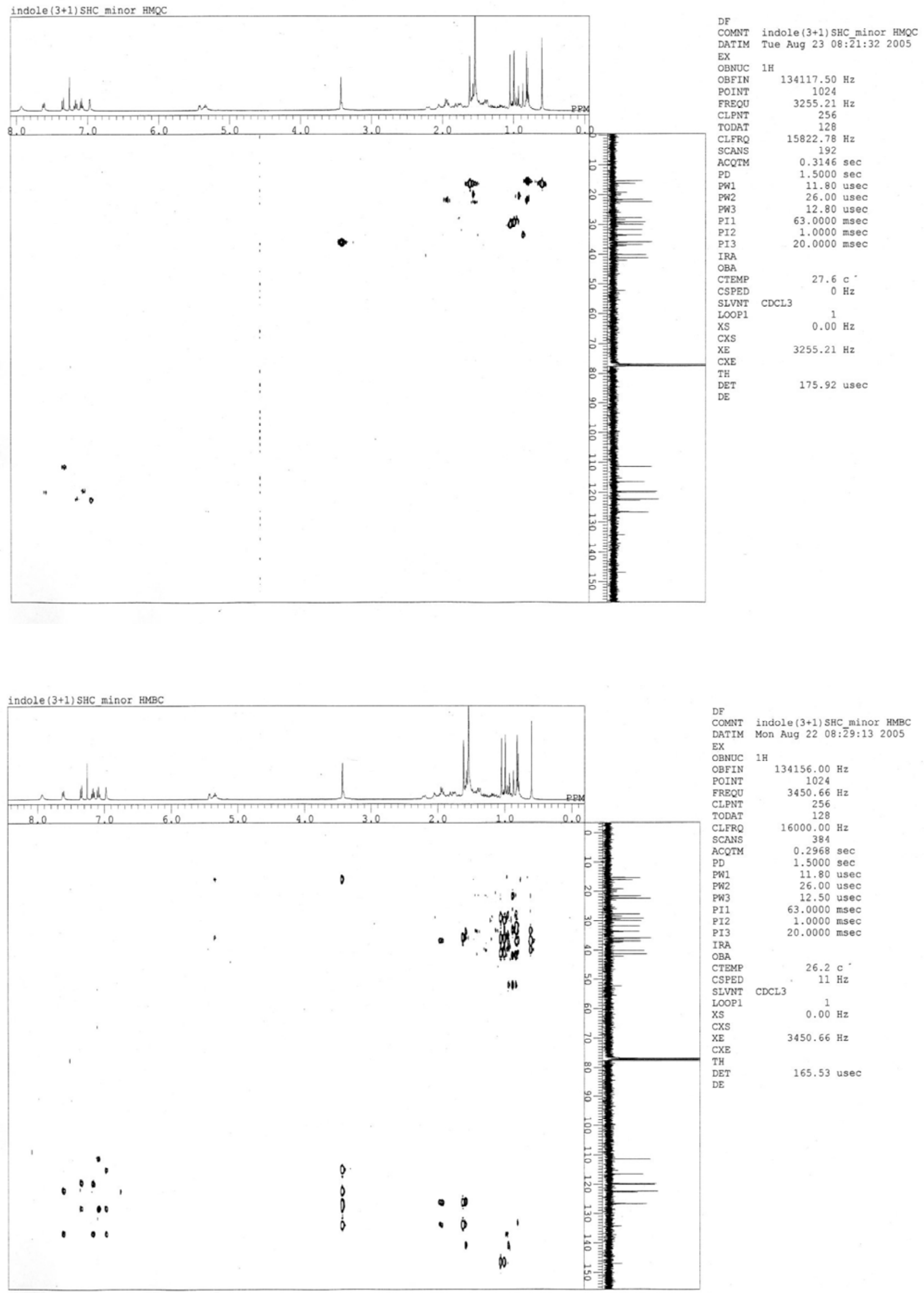
Minor product (7): NOE Experiment-1
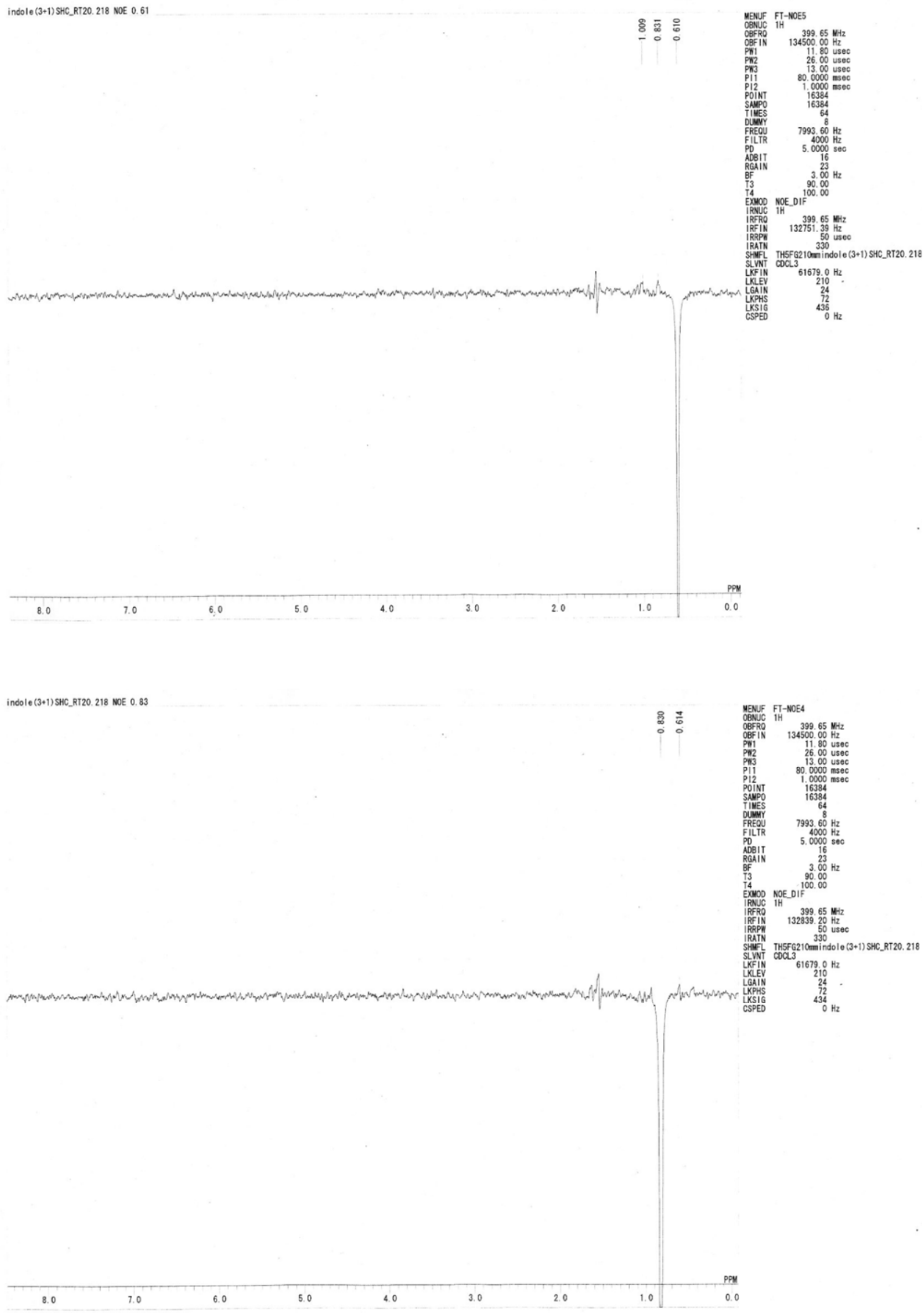
Minor product (7): NOE Experiment-2
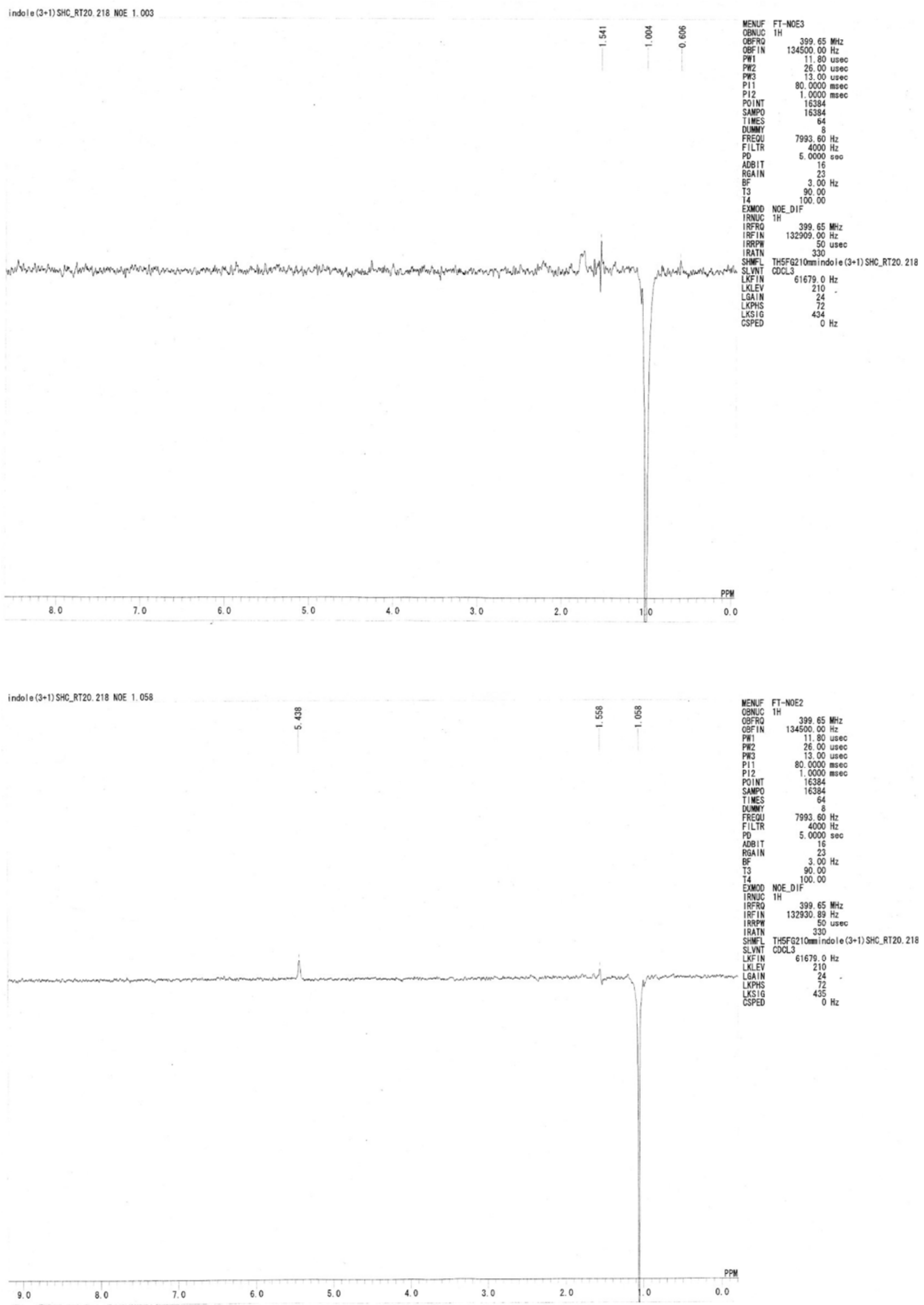
Minor product (7): NOE Experiment-3

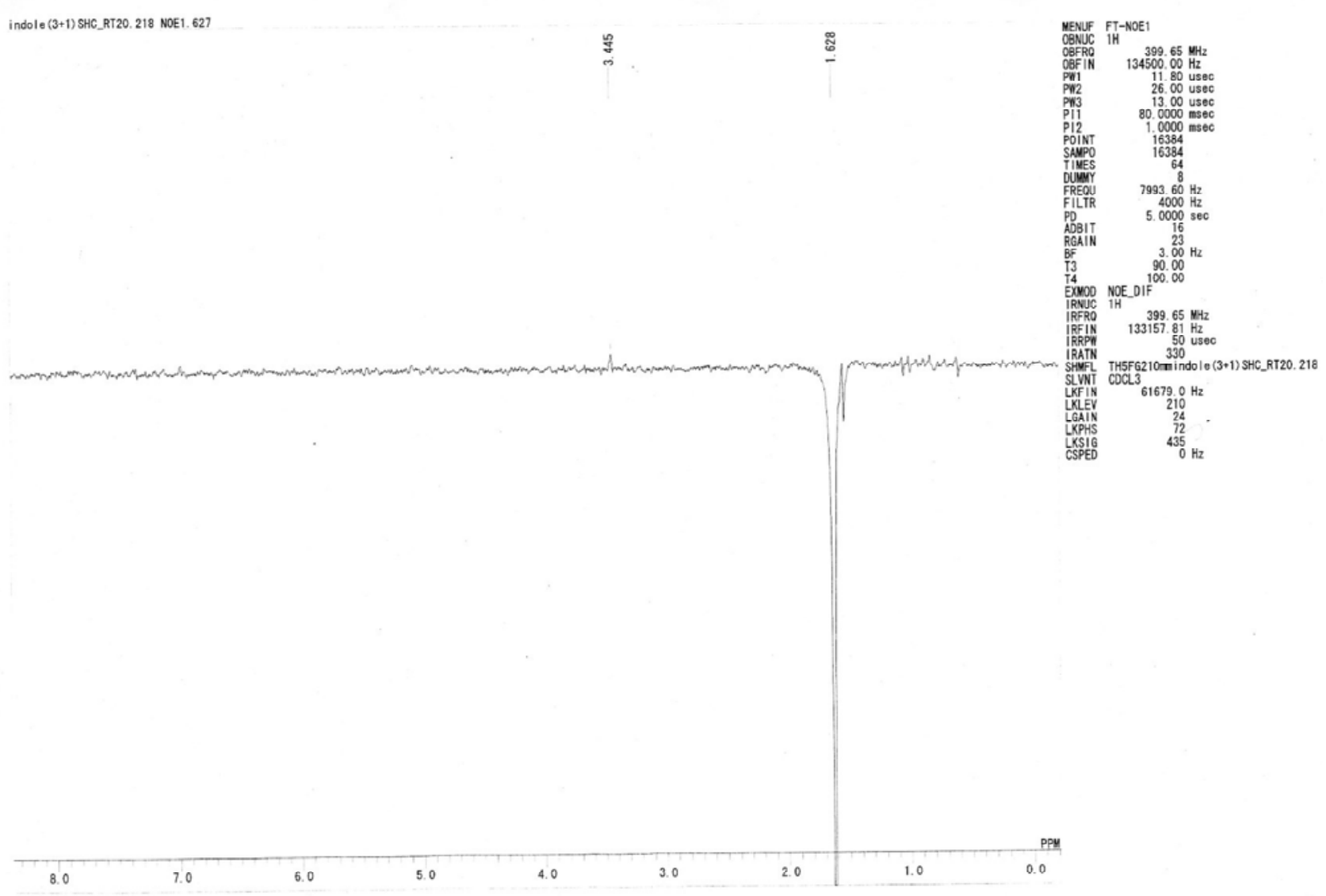

